\title{
Contemporary management of muscle-invasive bladder cancer
}

\author{
Marc A Dall'Era ${ }^{1}{ }^{,}$, Liang Cheng ${ }^{2}$, and Chong-Xian Pan ${ }^{3,4}$ \\ ${ }^{1}$ Department of Urology, University of California Davis, 4860 Y Street, Suite 3500, Sacramento, \\ CA 95817, USA \\ 2Department of Pathology and Laboratory Medicine, Indiana University School of Medicine, 350 \\ West 11th Street, Room 4010, Indianapolis, IN 46202, USA
}

${ }^{3}$ VA Northern California Health Care System, 10535 Hospital Way, Mather, CA 95655, USA

${ }^{4}$ Division of Hematology and Oncology, Department of Internal Medicine and Department of Urology, University of California-Davis Cancer Center, Sacramento, CA 95817, USA

\begin{abstract}
The current standard treatment for muscle-invasive nonmetastatic bladder cancer is neoadjuvant platinum-based chemotherapy followed by radical cystectomy. However, neoadjuvant chemotherapy is not widely accepted even with level 1 evidence. Adjuvant chemotherapy should be discussed if patients have not received neoadjuvant chemotherapy before surgery and have high-risk pathologic features. Although not considered standard of care, bladder-sparing therapy can be considered for highly selected patients and for those medically unfit for surgery. Even though there are no level 1 data, the treatment outcomes for highly select patients given bladdersparing therapy appear promising, with many patients retaining a functional bladder. Personalized chemotherapy is currently being actively pursued to target the underlying molecular changes and tailor to individual needs.
\end{abstract}

\section{Keywords}

bladder urothelial carcinoma; chemotherapy; cisplatin; personalized chemotherapy; targeted therapy

Carcinoma of the bladder of all histologic subtypes remains a major global health issue. The 2004 WHO World Cancer Report estimates that bladder cancer is the ninth most commonly diagnosed malignancy worldwide, accounting for 330,000 new cases annually [1,2]. Bladder cancer incidence varies dramatically from country to country, with the highest rates in North

\footnotetext{
(C) 2012 Expert Reviews Ltd
}

*Author for correspondence: Tel.: +1 9167342839 Fax: +1 9167348094 marc.dallera@ucdmc.ucdavis.edu.

For reprint orders, please contact reprints@expert-reviews.com

Disclaimer

The contents reported/presented within do not represent the views of the Department of Veterans Affairs or the United States Government.

Financial \& competing interests disclosure

This work is supported by the VA Career Development Award-2 (principal investigator: C-X Pan) and NCI Cancer Clinical Investigator Team Leadership Award (principal investigator: C-X Pan). C-X Pan is a co-founder of the Accelerated Medical Diagnostics that develops diagnostic tools using accelerator mass spectrometry. The authors have no other relevant affiliations or financial involvement with any organization or entity with a financial interest in or financial conflict with the subject matter or materials discussed in the manuscript apart from those disclosed.

No writing assistance was utilized in the production of this manuscript. 
America and Europe where urothelial carcinoma (UC) represents the most common histologic form encountered, and cigarette smoking continues to be the strongest risk factor of development of the disease [3]. It is estimated that, in the USA, there will be 73,510 new cases of bladder cancer in 2012 [4], with 14,880 deaths attributable to this disease. Bladder cancer mortality is less varied geographically, yet is highest in developed countries. Despite advances in diagnosis, treatment and molecular characterization of bladder tumors, the outcomes from bladder cancer treatment have not significantly improved over the last two decades. Bladder cancer can be clinically dichotomized by stage as either muscle invasive or non-muscle-invasive based on involvement of the detrusor muscle. Approximately $20 \%$ of tumors are muscle invasive at presentation, requiring aggressive and often systemic therapies. Clinical experience also clearly describes two general phenotypic variants of UC of the urinary bladder largely based on tumor grade, which is a well-established predictor of disease progression and bladder cancer-specific mortality [5]. High-grade bladder tumors harbor significant risks for recurrence, local invasion and metastasis, and are primarily responsible for the high degree of mortality associated with UC. This review will focus on the contemporary management of high-grade muscle-invasive UC of the bladder.

\section{Objectives}

To review the current literature on treatment options and outcomes for muscle-invasive localized UC.

\section{Methods}

We searched MEDLINE, EMBASE, the Cochrane Central Registry of Controlled Trials, the Cochrane Methodology Registry, and the Cochrane Database of Systematic Reviews. All searches were performed with the assistance of a certified medical librarian. The search terms included bladder cancer, urinary bladder neoplasms, urothelial carcinoma, transitional cell carcinoma, muscle invasive, not nonmuscle invasive, advanced, and myoinvasive. No language restrictions were imposed. The reference lists from retrieved articles were examined to identify additional relevant studies. The abstracts from all retrieved citations were reviewed to determine their eligibility for inclusion in this paper and full-length articles were then reviewed for final selection. For the results section of this paper, preference was given to studies describing results from randomized data and meta-analyses. Clinical trials and population-based observational studies comparing treatment strategies for primarily high-grade muscle-invasive bladder cancer were sought. Studies focusing on superficial UC, regardless of grade, including primarily carcinoma in situ were excluded. To meet inclusion criteria, studies had to provide a comprehensive description of the demographic and disease characteristics of the patients at the time of diagnosis, including data on clinical or pathologic stage and tumor grade.

\section{Results}

\section{Radical cystectomy}

Radical cystectomy has long been considered the 'standard of care' for muscle-invasive UC. Radical cystectomy typically includes complete surgical removal of the bladder and prostate in men and the bladder, uterus and all or part of the vagina in women [6]. Pelvic lymphadenectomy is often routinely performed and may provide both diagnostic and therapeutic benefit as discussed later. Contemporary series describe 5-year overall survival rates of 59-66\% with radical cystectomy alone with recurrence-free survival ranging from 62 to $68 \%[7,8]$. Perioperative mortality from radical cystectomy has decreased over time from nearly $20 \%$ in the 1960 s to $3-7 \%$ in contemporary series [7,9]. Disease-specific and 
overall survival for patients with high-grade UC are closely linked with pathologic disease stage, with 5-year survival dropping to $26 \%$ for men with positive lymph nodes [8].

Several studies suggest that surgical techniques, including extent of lymph node dissection, may influence outcomes after radical cystectomy [10-12]. Herr et al. performed a secondary analysis of data obtained from a randomized trial comparing neoadjuvant chemotherapy with surgery alone in patients with invasive UC. Multivariate analyses showed that negative surgical margin status and number of lymph nodes removed $(>10)$ were associated with improved survival in both treatment arms [12]. Analyzing data from the Memorial SloanKettering institutional database of 1121 cases of radical cystectomy with lymph node dissection, Koppie et al. showed that, after adjustment with covariates, a continuous positive correlation between the number of lymph nodes removed and survival after radical cystectomy without a plateau was observed [11]. This study suggests that there was no optimal cutoff lymph node number, and it is probable that the extent and meticulousness of the dissection are critical factors for improved oncologic outcomes. Although it remains to be shown if the role of lymph node dissection in bladder cancer is more diagnostic or therapeutic, extended dissection with radical cystectomy provides more accurate pathologic staging for determining prognosis or the need for adjuvant treatment or clinical trial eligibility and appears to correlate with improved survival after surgery. A Southwest Oncology Group (SWOG) collaborative clinical trial comparing limited to extended lymph node dissection at the time of cystectomy is currently underway. Improved outcomes from cystectomy have also been associated with high-volume centers, suggesting that surgeon and hospital experience are important in managing UC [13].

Recent experiences with laparoscopic and robotic radical cystectomy and lymphadenecotmy have been described. Although reported median follow-up remains short, these approaches appear to have similar morbidities and functional outcomes to open approaches [14]. Ultimately, minimally invasive approaches may prove effective in reducing pain and operative blood loss associated with this operation.

Data from retrospective surgical series show that despite aggressive and often early intervention, many patients with muscle-invasive bladder cancer treated by surgery alone still remain at considerable risk for recurrence and death from bladder cancer [7]. The majority of these recurrences occur within 3 years of surgery and $75 \%$ of these patients fail with distant metastases [7]. Radical cystectomy achieves excellent local control; however, many patients present with clinically advanced and likely micro-metastatic disease at the time of diagnosis, and these data have prompted investigators to explore the abilities of neoadjuvant or adjuvant therapies to improve these outcomes.

\section{Radiation therapy}

The role of radiation therapy in the management of muscle-invasive bladder cancer remains unknown. The majority of studies identified for this review describe results with integrated radiation in a multimodal approach to bladder cancer therapy either neoadjuvantly before planned cystectomy or as part of a bladder-preserving protocol. Few studies compared radiation followed by surgery to radiation alone for treatment of bladder cancer. In 1982, Bloom et al. published results of a clinical trial comparing neoadjuvant radiotherapy before cystectomy to definitive radiotherapy followed by salvage cystectomy for progressive disease in 189 men with advanced UC [15]. The patients in the neoadjuvant arm received 40 Gy of whole pelvis radiation 4 weeks prior to planned radical cystoprostatectomy, while the definitive radiotherapy arm received $40 \mathrm{~Gy}$ to the pelvis with a 20-Gy boost to the bladder and perivesical tissue. With an intent-to-treat analysis, the 10-year cancer-specific mortality ratio was 1.77 (95\% CI: 0.92-3.4) in favor of neoadjuvant radiation followed by surgery over the radiation with delayed salvage cystectomy [15]. Later, Sell et al. published their 
results of a randomized trial comparing 40-Gy neoadjuvant radiotherapy followed by radical cystectomy to $60 \mathrm{~Gy}$ of radiation alone in 183 men with T2-T4 bladder cancer. As with the previous study, the authors reported no significant overall survival advantage at 5 years for men treated in the surgery arm (hazard ratio [HR]: 1.39; 95\% CI: 0.72-2.68) [16]. A total of 22 men $(25 \%)$ in the cystectomy arm did not undergo surgery while 27 men $(28 \%)$ in the radiation arm underwent salvage cystectomy. Both of these early studies were relatively small with fairly wide confidence intervals, probably making them underpowered to detect modest differences in survival. A pooled meta-analysis, including three studies comparing neoadjuvant radiation to radiation alone with salvage cystectomy, showed $36 \%$ overall survival at 5 years in the neoadjuvant/cystectomy group compared with $20 \% 5$-year survival in the radiation arm, with an overall odds ratio of 2.17 (95\% CI: 1.39-3.38) favoring neoadjuvant radiation and early surgery. It is important to note that these studies contained higher risk patients than in contemporary surgical series and probably show a survival advantage with early surgery compared with radiation alone for bladder cancer, even with delayed cystectomy.

A nonrandomized study by Skinner et al. studied the efficacy of neoadjuvant radiation before cystectomy compared with cystectomy alone in 197 consecutive patients and showed no effect on time to recurrence or overall survival with $1600 \mathrm{rad}$ of radiation given for 4 days prior to surgery [17]. A more contemporary series by Smith et al. reported results of a SWOG trial comparing neo-adjuvant radiation therapy followed by cystectomy compared with cystectomy alone and, again, showed no improvement in survival by the addition of radiation therapy before surgery [18]. Indeed, the toxicities associated with radiation in these trials were not trivial and must be carefully weighed in treatment decisions. Given these data, radiation therapy has no determined role as monotherapy for muscle-invasive bladder cancer or neoadjuvantly before cystectomy. Radiotherapy, however, has been integrated into several multimodal bladder-sparing protocols, as will be discussed subsequently.

\section{Systemic chemotherapy}

Despite excellent control with surgery for localized disease, a substantial number of patients recur after radical cystectomy alone, particularly patients with more advanced disease at the time of diagnosis. As has been found with other solid tumors, systemic chemotherapy offers the possibility of disease consolidation prior to extirpative surgery as well as the potential to treat clinically undetected micrometastatic disease to improve survival after surgery. Multiple studies have demonstrated the effects of cytotoxic chemotherapy for UC [19,20]. For years, researchers have investigated the efficacy of adjuvant therapies on bladder cancer outcomes after radical cystectomy and a number of single-agent therapies were identified that demonstrated some efficacy $[21,22]$.

Neoadjuvant chemotherapy-A 1999 meta-analysis sponsored by the British Medical Research Council (MRC) combined data from four randomized trials including 479 patients comparing single-agent cisplatin in the neoadjuvant setting for invasive bladder cancer. With 301 deaths and median follow-up of 3 years, overall survival was assessed and no statistically significant survival benefit was found with cisplatin monotherapy before surgery with an overall HR of 1.02 (95\% CI: 0.81-1.26) [23]. Given a lack of clear benefit with single-agent therapy, combination chemotherapy has also been investigated and shown to have a more promising effect on bladder cancer (Table 1) [24]. In 1999, the MRC/European Organization for Research and Treatment of Cancer (EORTC) published results of a neoadjuvant trial using combination chemotherapy in 976 patients with high-grade muscleinvasive and locally advanced bladder cancer [25]. Patients were randomized to receive the three-drug neoadjuvant regimen of cisplatin, methotrexate and vinblastine (CMV) followed by surgery or radiation versus surgery or radiation alone. Of the cohort, 285 patients 
underwent cystectomy with or without neoadjuvant chemotherapy. Overall survival showed a trend towards, but nonstatistically significant, advantage with neoadjuvant chemotherapy (HR: $0.85 ; 95 \% \mathrm{CI}: 0.71-1.02 ; \mathrm{p}=0.075$ ) with a $5.5 \%$ absolute difference in 3 -year survival between the groups. A recent update of this trial with a median follow-up of 8 years shows a survival advantage with neoadjuvant chemotherapy (relative risk [RR]: 0.88 ; 95\% CI: $0.72-$ $0.99 ; p=0.037$ ) [26]. Of note, the complete pathologic response (pT0) rate was $32.5 \%$ in the chemotherapy arm compared with $12.3 \%$ in the control arm. In an effort to increase the power to detect small yet significant differences, an Advanced Bladder Cancer metaanalysis published in 2003 combined data on 2688 patients from ten randomized neoadjuvant chemotherapy bladder cancer trails [1,27]. With some heterogeneity, all trials used platinum-based chemotherapy either alone or in combination. When analyzing the patients from the six trials incorporating combination platinum-based chemotherapy, the study noted a combined HR of 0.87 (95\% CI: 0.78-0.97) in favor of neoadjuvant chemotherapy for overall survival with an absolute survival benefit of $5 \%$ at 5 years [27].

The SWOG trial 87-10 was designed to further evaluate the efficacy of neoadjuvant combination chemotherapy in a randomized fashion prior to surgical management of invasive bladder cancer [28]. Patients with muscle-invasive (T2-T4a), nonmetastatic bladder cancer who were candidates for radical cystectomy were randomly assigned to receive surgery alone or three cycles of methotrexate, vinblastine, doxorubicin and cisplatin (MVAC) followed by radical cystectomy. Over an 11-year period between 1987 and 1998, this trial enrolled 317 patients with bladder cancer who had no prior systemic chemotherapy or radiation therapy. With a primary end point of overall survival and a median follow-up of 8.7 years, this study demonstrated an improved survival for patients receiving neoadjuvant chemotherapy, with a median survival of 77 months compared with 46 months in the cystectomy-alone arm $(\mathrm{p}=0.06)$ [28]. This yielded a HR of 1.33 (95\% CI: 1.00-1.76) for risk of death for men not receiving combination therapy. Importantly, the chemotherapy delivered in this trial was generally well tolerated and did not seem to impair a patient's chances of undergoing surgery. As with the MRC trial, the effect of chemotherapy on disease stage was described and found to be significant. Pathologic T0 (no residual disease) rates were significantly higher in the chemotherapy arm, where $38 \%$ of men were pT0 at the time of cystectomy compared with $9 \%$ in the surgery-alone group. The SWOG trial further went on to show that, not surprisingly, men who were pT0 at the time of surgery in either arm had the best outcomes with an $85 \%$ survival at 5 years.

A combined analysis of two Nordic cystectomy trials with neo-adjuvant combination cisplatin-based chemotherapy showed a HR of 0.8 for overall survival (95\% CI: $0.64-0.99$; $\mathrm{p}=0.049$ ) [29]. Neither trial alone showed a statistically significant treatment effect with neoadjuvant chemotherapy on overall survival, but as with the MRC/EORTC trial, neither was powered to detect a modest effect. Taken together, these data suggest a positive, yet likely modest, effect of neoadjuvant cisplatin-based combination chemotherapy on bladder cancer. With the level 1 evidence from this and the SWOG trial, this should be considered standard of care for all patients with muscle-invasive UC in the absence of any clear contraindications.

These trials clearly demonstrate that patients with bladder cancer respond varyingly to systemic chemotherapy. So far, no reliable method is available to distinguish responders from nonre-sponders, despite the development of new technologies to prospectively identify chemoresistance before chemotherapy is initiated (reviewed in [30]). Not surprisingly, patients who responded to the chemotherapy had the best long-term survival after surgery, and the ability to identify these patients upfront may limit any associated toxicities to those who have the most to gain from systemic chemotherapy while identifying patients best suited for other agents or novel clinical trials. 
Adjuvant chemotherapy-At risk of overtreating some men with chemotherapy, several authors argue for immediate cystectomy followed by selective adjuvant chemotherapy for patients found to have high-risk pathologic features [31]. Indeed, delay of radical cystectomy has been shown to correlate with decreased survival and may mitigate the advantages of neoadjuvant therapy [32]. Table 2 lists the included studies utilizing adjuvant combined chemotherapy for patients with high-risk features after radical cystectomy. Freiha et al. described their single-institution experience with adjuvant chemotherapy for locally advanced or lymph node metastatic bladder cancer after radical cystectomy [33]. The trial randomized 55 patients with locally advanced bladder cancer to receive cisplatin-based combination chemotherapy or standard surveillance after radical cystectomy. Although no overall survival advantage was demonstrated, there was a significant increase in time to progression for patients randomized to receive adjuvant chemotherapy (37 vs 13 months; $\mathrm{p}<$ 0.01 ) [33]. A recent meta-analysis pooled data of 491 patients from six randomized adjuvant chemotherapy trials after radical cystectomy [34]. All included trials used cisplatin-based chemotherapy, one as single agent and the rest in combination with other agents. The majority of patients in these trials had high-grade and pathologically advanced (T3-T4) disease at cystectomy with approximately $35 \%$ having lymph node-positive disease. The HR for overall survival was 0.75 (95\% CI: $0.6-0.96$ ) in favor of adjuvant chemotherapy with an absolute improvement in disease-free survival of $12 \%$. On review of the included trials, however, nearly $25 \%$ of patients randomized to adjuvant chemotherapy did not receive the additional therapy, suggesting that many patients at high risk for recurrence did not get additional therapy for a variety of reasons. In general, it has been demonstrated that patients are more likely to tolerate the effects of chemotherapy before major surgery, and any impairment in renal function after surgery may limit the use of certain effective agents and dosages [35]. A recent retrospective study involving 3947 patients from 11 centers showed that adjuvant chemotherapy is independently associated with improved overall survival (HR: 0.83 ; 95\% CI: $0.72-0.97 ; \mathrm{p}=0.017$ ), especially in the highest-risk quintile (HR: $0.75 ; 95 \%$ CI: $0.62-0.90 ; p=0.002$ ) [36]. Currently, adjuvant chemotherapy is recommended for patients not receiving prior chemotherapy with high-risk pathologic features found at the time of surgery, most notably pathologic stage $\geq T 3 \mathrm{a}$ or lymph node involvement.

No chemotherapy is without potential side effects and toxicities, and the decision to use chemotherapy must be made after careful discussion and counseling between physicians and patients based on solid scientific evidence. MVAC treatment has been commonly associated with leukopenia, mucositis, nausea and vomiting that is significantly higher than with single-agent cisplatin [37]. A randomized trial comparing MVAC with cisplatin alone in patients with metastatic bladder cancer reported a $4 \%$ therapy-related mortality with MVAC, and only $24 \%$ of the patients received full-dose MVAC without dosage modifications, which is clearly not inconsequential [37]. These data have led investigators to study other regimens with similar activity against bladder cancer but less toxicity. Several Phase II bladder cancer trials showed anti-tumor activity with cisplatin in combination with gemcitabine [31,38]. A large, multicenter randomized trial in patients with locally advanced or metastatic bladder cancer was performed comparing the combination of gemcitabine and cisplatin with MVAC [31,39]. This trial did not show significant difference in overall response rate (49 vs $46 \%$ ), time to progression ( 7.4 vs 7.4 months), and median survival (13.8 vs 14.8 months) between the two regimens, yet it showed a much improved safety profile with gemcitabine and cisplatin $[31,39]$. The most significant toxicities associated with this two-drug regimen appear to be neutropenia and thrombocytopenia; more patients received GM-CSF in the gemcitabine-cisplatin arm, perhaps accounting for some of the effect seen. Although this trial was designed as a superiority trial for the gemcitabine-cisplatin combination for metastatic bladder cancer (and was negative), we can conclude that there were no demonstrable survival differences between regimens in this study. Although no level 1 evidence favors gemcitabine-cisplatin over MVAC in the neoadjuvant setting, these data 
have led many clinicians to favor the gemcitabine-cisplatin combination for neoadjuvant or adjuvant therapy in bladder cancer given the demonstration of general efficacy with apparent reduced morbidity. Data suggest that high-dose-intensity MVAC administered with GM-CSF is better tolerated than standard MVAC, allowing for less toxicity and fewer missed cycles [40]. There are currently two trials using dose-dense chemotherapy for patients with muscle-invasive bladder cancer, including one in the neoadjuvant setting [101].

Despite level 1 evidence suggesting a survival advantage with combination neoadjuvant chemotherapy before radical cystectomy, the majority of contemporary patients with muscle-invasive disease do not receive this treatment [41]. Based on a recent analysis of 40,388 patients with muscle-invasive (stages II-IV) bladder cancer in 2003-2007 from the National Cancer Database, there is an increased acceptance for neoadjuvant chemotherapy. Even so, only 13\% of patients received this therapy in 2007, compared with 6\% in 2003 $[42,43]$. It remains unclear why there has not been wider acceptance of this treatment paradigm for muscle-invasive disease. Perhaps many surgeons and patients are not willing to accept delaying cystectomy and assuming the risks of chemotherapy for a modest survival benefit. With the clear inaccuracies of clinical staging and until we can identify those patients that are likely to benefit or respond to systemic therapy, overtreatment remains a concern. We continue to advocate that all patients with muscle-invasive clinically localized disease be offered neoadjuvant chemotherapy followed by radical cystectomy and lymphadenectomy as standard of care for this disease. Future research efforts focused on identifying predictors of individual patient response to chemotherapy may have a profound impact on the treatment of invasive UC [30]. Gandour-Edwards et al. described HER2 expression by immunohistochemistry in advanced (stage III or IV) bladder cancer and correlated expression patterns with outcomes including response to cytotoxic chemotherapy [44]. Although staining did not correlate with response to paclitaxel-based chemotherapy or progression-free survival, positive staining was associated with improved overall survival [44]. A more recent publication also showed that HER2 overexpression is associated wtih gene amplification in approximately 5\% of tumors [45]. A Phase III study evaluated the value of p53 status at the time of cystectomy for predicting disease recurrence, overall survival and benefit from adjuvant chemotherapy [46]. This trial failed to demonstrate a correlation between p53 inactivation and any of the study end points; however, there were a number of accrual and methodological limitations, including the fact that p53 status may not be best assessed with immunohistochemistry as performed in the study. Ongoing studies will provide improved molecular signatures of bladder cancer to provide patient tumor-directed targeted therapies, many of which may ultimately be administered by the urologic oncologist.

\section{Bladder-sparing therapies}

As an alternative to the standard of care for muscle-invasive bladder cancer, certain noncystectomy, bladder-sparing protocols should be offered to patients with significant comorbidities, because of patient preferences, or in highly selected patients (Table 3 ). It must be kept in mind, however, that many patients with poor performance status deemed 'unfit' for surgery are also unlikely to tolerate aggressive systemic chemotherapy. Bladder preservation protocols may have a curative or palliative intent based on the overall goals of therapy for the patient. Even though there is no randomized clinical trial comparing cystectomy and bladder-sparing therapy, mounting evidence suggests that carefully managed patients may have extended survival and maintain quality of life with intact bladders. In a nonrandomized study, Herr et al. described the results of 99 selected patients with muscle-invasive UC choosing to follow their bladder tumors after restaging transurethral resection (TUR) showed either no residual or only superficial residual disease [47]. After a minimum follow-up of 10 years, 75 patients (76\%) were alive while 24 patients 
(24\%) were dead of disease. Of the 75 patients surviving at least 10 years, 57 had their bladders intact, suggesting that some carefully selected patients, even with muscle-invasive disease, could experience long-term survival with only local resection.

Given the known activity of platinum-based chemotherapy against bladder cancer, multiple centers have gained considerable experience with the bladder-preserving multimodal approach to the management of muscle-invasive high-grade UC [48-54]. Solsona et al. recently reported the results of a Phase II nonrandomized bladder preservation study evaluating the efficacy of TUR with adjuvant systemic chemotherapy for select patients with muscle invasive UC [49]. Specific inclusion criteria included nonmetastatic muscle-invasive UC with positive residual biopsies of the tumor bed performed at the time of TUR. Patients meeting these criteria were offered three courses of systemic chemotherapy or immediate radical cystectomy. Patients getting chemotherapy were followed with repeat bladder biopsies initially every 3 months after treatment. With a median follow-up of 45 months, 40 patients $(53 \%)$ had an objective response by TUR, and 5-year and 10-year overall cancerspecific survival with bladder preservation was $64.5 \%$ and $59.8 \%$, respectively [49]. A separate study assessed the value of combined chemotherapy with concurrent radiotherapy after complete TUR in 53 patients with T2-T4 bladder cancer [51]. After complete TUR of the bladder tumor, patients were treated with combination platinum-based systemic chemotherapy and whole pelvis external beam radiotherapy. Radical cystectomy was offered to all patients not achieving a complete response by TUR after this initial treatment. After a median follow-up of 4 years, $53 \%$ of participants were alive, $45 \%$ without evidence of disease. Fifteen patients went on to receive radical cystectomy, giving an overall rate of bladder preservation of 58\% for the duration of the study [51].

Rodel et al. reported their series of combined modality therapy with the goal of bladder preservation for muscle-invasive bladder cancer [50]. A total of 126 patients were treated with pelvic radiation alone after TUR while 289 received concomitant pelvic radiation and systemic chemotherapy. Patients underwent repeat TUR 6 weeks after initial therapy with $72 \%$ achieving a complete detectable response. In total, $20 \%$ of patients ultimately went on to receive salvage cystectomy for residual invasive or recurrent disease. This group of initial nonresponders demonstrated 5-year and 10-year cancer-specific survival of 50\% and 45\%, respectively, suggesting that many patients were successfully salvaged with surgery after failed radiation with or without combination chemotherapy. Patients with initial complete response also demonstrated significantly better metastasis-free survival than those who did not. Survival was significantly better for patients with local recurrence after initial complete response compared with those failing immediately. Overall 5-year and 10-year survival for the entire cohort was 51 and $31 \%$, respectively. Only $2 \%$ of patients required cystectomy because of contracted bladder [50]. It must be stressed that bladder-preserving strategies for muscle-invasive UC are not considered standard of care and these data must be interpreted with caution. These reports contain carefully selected patients who are not comparable to those in neoadjuvant or adjuvant radical cystectomy trials.

As previously discussed, the neoadjuvant chemotherapy trial SWOG 87-10 demonstrated that patients with no residual disease in the specimen (pT0) after radical cystectomy and neoadjuvant chemotherapy achieved the longest overall and disease-specific survival. This generates questions regarding the need for cystectomy if chemosensitive tumors were completely removed with local resection and systemic chemotherapy. It remains to be determined whether the pT0 status can be reliably ascertained with TUR or biopsy alone after neoadjuvant therapy. The Phase II SWOG trial 02-19 was designed to address this question [53]. Patients with T2-T4a bladder cancers were included and received three cycles of combination chemotherapy followed by repeat bladder biopsy. Patients with a complete clinical response were offered immediate cystectomy or bladder preservation with regular 
cystoscopic surveillance. A total of 34 out of 74 patients (46\%) had a complete clinical response based on post-treatment TUR and ten patients elected to undergo immediate cystectomy despite no demonstrable disease. The study found a concerning high rate of persistent cancer (six out of ten patients) after radical cystectomy in patients presumed to have a complete response by TUR alone. With a median follow-up of 22 months, patients with a complete clinical response demonstrated $75 \%$ overall survival compared with $59 \%$ for the entire cohort; however, these findings prompted the authors to conclude that radical cystectomy must still be pursued after chemotherapy for definitive local control of bladder cancer given the high residual tumor rate after radical cystectomy in patients with presumed complete clinical responses.

\section{Expert commentary}

Muscle-invasive UC requires multidisciplinary care that should include coordinated efforts from medical oncology, urology and sometimes radiation oncology. Fully staging workups should be performed to rule out lymph node and distant metastasis. There is level 1 evidence supporting the use of platinum-based combination neoadjuvant chemotherapy. However, it is not widely accepted in practice. Radical cystectomy is still the mainstay of therapy. Those patients who do not receive neoadjuvant chemotherapy should receive adjuvant chemotherapy that can possibly improve the overall survival. In highly selected or medically unfit patients, bladder-sparing therapy can be performed with comparable outcomes.

\section{Five-year view}

The treatment outcomes of locally advanced bladder UC have not changed over the last three decades. Bladder cancer mortality can possibly be reduced by optimizing the currently available therapy and improving new drug development. Current treatment strategies for muscle-invasive bladder cancer still take the one-formula-for-all approach; however, every patient is different. We expect to see that personalized chemotherapy will be intensively studied in bladder cancer in the next 5 years.

One approach of personalized therapy is to tailor chemotherapy based on the cancer genetic changes. ERCC1 and $R R M 1$ are two genes involved in DNA repair and deoxyribonucleotide production. They have been studied to help select platinum and gemcitabine chemotherapy in non-small-cell lung cancer [55-57]. Both genes are also involved in bladder UC. Low ERCC1 expression is associated with higher progression-free survival than those with high ERCC1 expression levels after adjuvant cisplatin-based therapy (5-year PFS: 45 vs 70\%; HR: $0.52 ; p=0.03$ ) [58]. Young patients with high RRM1 expression had longer median overall survival of 10.6 years compared with 1.6 years in older patients $(p=0.001)$ [59], suggesting that it may have a prognostic role in predicting survival and selecting adjuvant chemotherapy. High hENT1 expression has also been associated with response to gemcitabine as it is a major cell membrane nucleotide transporter [60]. Several other groups are also studying the combination of genes and miRNA panels [61-63]. However, prospective randomized clinical trials are required to establish the value of these genes in personalized chemotherapy for bladder cancer.

Trials in the neoadjuvant setting are critical to provide more personalized targeted therapy for bladder cancer. Using a coexpression extrapolation (COXEN) approach, researchers have used cell lines in vitro to identify active therapeutic compounds with high throughput against bladder cancer [64]. This approach also allows for identification of specific gene expression signatures for predicting response to chemotherapy [64]. A group of researchers at the University of California, Davis (CA, USA) is developing an in vivo in-patient test under physiological conditions to identify chemoresistance before bladder cancer patients receive therapeutic chemotherapy. This uses microdosing and accelerator mass spectrometry 
to measure platinum-induced DNA damage, a critical intermediate in platinum-induced cell death. The hypothesis, which is supported by preliminary data $[65,66]$, is that cancer cells with low platinum-induced DNA damage will survive chemotherapy and thus differentiate nonresponders from responders. Accelerator mass spectrometry measures ${ }^{14} \mathrm{C}$ at the $10^{-18}$ mole level. When ${ }^{14} \mathrm{C}$-labeled platinum is used for the test, platinum-induced DNA damage can be measured by determining the ${ }^{14} \mathrm{C}$ content of genomic DNA. Because of the high sensitivity, this test can be performed after patients receive one nontoxic microdose of ${ }^{14} \mathrm{C}$ labeled drug. A clinical trial is currently ongoing to determine the clinical application of this test [102]. There are unmet needs for early-stage muscle-invasive bladder cancer clinical trials. As previously mentioned, the SWOG S011 is currently accruing and will better define the role of extended lymph node dissection at the time of radical cystectomy for muscleinvasive bladder cancer. Other ongoing trials are evaluating dose-dense chemotherapy in the neoadjuvant setting for muscle-invasive disease.

Another approach to improve the treatment outcomes of UC is to develop novel therapeutics. As the underlying pathophysiological process in UC is elucidated, more targeted therapy will be tested in UC. Recently, a bladder cancer-targeting ligand has been developed that can specifically bind to both human and dog bladder cancer cells in vitro and in vivo [67-69]. Nanomicelles loaded with a chemotherapeutic (paclitaxel) and decorated with these cancer-targeting ligands could prolong cancer control and overall survival in a mouse model carrying patient-derived xenografts directly developed from clinical bladder cancer patient specimens [Pan C-X et al., Unpublished Data] This targeting nanoparticle is expected to go into a Phase I trial within the next 3 years.

\section{References}

1. The World Cancer Report - the major findings. Cent Eur J Public Health. 2003; 11(3):177-179. [PubMed: 14514174]

2. Stewart, BW. World Cancer Report 2003. IARC Press; Lyon, France: 2004.

3. Johansson SL, Cohen SM. Epidemiology and etiology of bladder cancer. Semin Surg Oncol. 1997; 13(5):291-298. [PubMed: 9259084]

4. Siegel R, Naishadham D, Jemal A. Cancer statistics, 2012. CA Cancer J Clin. 2012; 62(1):10-29. [PubMed: 22237781]

5. Wu XR. Urothelial tumorigenesis: a tale of divergent pathways. Nat Rev Cancer. 2005; 5(9):713725. [PubMed: 16110317]

6. Stein JP, Skinner DG. Surgical atlas. Radical cystectomy. BJU Int. 2004; 94(1):197-221. [PubMed: 15217471]

7. Stein JP, Lieskovsky G, Cote R, et al. Radical cystectomy in the treatment of invasive bladder cancer: long-term results in 1,054 patients. J Clin Oncol. 2001; 19(3):666-675. [PubMed: 11157016]

8. Madersbacher S, Hochreiter W, Burkhard F, et al. Radical cystectomy for bladder cancer today - a homogeneous series without neoadjuvant therapy. J Clin Oncol. 2003; 21(4):690-696. [PubMed: 12586807]

9. Ghoneim MA, Abdel-Latif M, El-Mekresh M, et al. Radical cystectomy for carcinoma of the bladder: 2,720 consecutive cases 5 years later. J Urol. 2008; 180(1):121-127. [PubMed: 18485392]

10. Leissner J, Hohenfellner R, Thuroff JW, Wolf HK. Lymphadenectomy in patients with transitional cell carcinoma of the urinary bladder; significance for staging and prognosis. BJU Int. 2000; 85(7): 817-823. [PubMed: 10792159]

11. Koppie TM, Vickers AJ, Vora K, Dalbagni G, Bochner BH. Standardization of pelvic lymphadenectomy performed at radical cystectomy: can we establish a minimum number of lymph nodes that should be removed? Cancer. 2006; 107(10):2368-2374. [PubMed: 17041887] 
12. Herr H, Lee C, Chang S, Lerner S. Standardization of radical cystectomy and pelvic lymph node dissection for bladder cancer: a collaborative group report. J Urol. 171(5):823-1828. discussion 1827-1828 (2004).

13. Goossens-Laan CA, Gooiker GA, Van Gijn W, et al. A systematic review and meta-analysis of the relationship between hospital/surgeon volume and outcome for radical cystectomy: an update for the ongoing debate. Eur Urol. 2011; 59(5):775-783. [PubMed: 21310525]

14. Styn NR, Montgomery JS, Wood DP, et al. Matched comparison of robotic-assisted and open radical cystectomy. Urology. 2012; 79(6):1303-1309. [PubMed: 22516354]

15. Bloom HJ, Hendry WF, Wallace DM, Skeet RG. Treatment of T3 bladder cancer: controlled trial of pre-operative radiotherapy and radical cystectomy versus radical radiotherapy. Br J Urol. 1982; 54(2):136-151. [PubMed: 7044462]

16. Sell A, Jakobsen A, Nerstrom B, Sorensen BL, Steven K, Barlebo H. Treatment of advanced bladder cancer category T2 T3 and T4a. A randomized multicenter study of preoperative irradiation and cystectomy versus radical irradiation and early salvage cystectomy for residual tumor DAVECA protocol 8201 Danish Vesical Cancer Group. Scand J Urol Nephrol Suppl. 1991; 138:193-201. [PubMed: 1785004]

17. Skinner DG, Lieskovsky G. Contemporary cystectomy with pelvic node dissection compared to preoperative radiation therapy plus cystectomy in management of invasive bladder cancer. J Urol. 1984; 131(6):1069-1072. [PubMed: 6726903]

18. Smith JA Jr, Crawford ED, Paradelo JC, et al. Treatment of advanced bladder cancer with combined preoperative irradiation and radical cystectomy versus radical cystectomy alone: a Phase III intergroup study. J Urol. 157(3):805-807. discussion 807-808 (1997). [PubMed: 9072571]

19. Wallace DM, Raghavan D, Kelly KA, et al. Neo-adjuvant (pre-emptive) cisplatin therapy in invasive transitional cell carcinoma of the bladder. Br J Urol. 1991; 67(6):608-615. [PubMed: 2070206]

20. Coppin CM, Gospodarowicz MK, James K, et al. Improved local control of invasive bladder cancer by concurrent cisplatin and preoperative or definitive radiation. The National Cancer Institute of Canada Clinical Trials Group. J Clin Oncol. 1996; 14(11):2901-2907. [PubMed: 8918486]

21. Nanus DM, Bosl GJ. Systemic chemotherapy in the management of bladder cancer. Cancer Treat Res. 1989; 46:143-158. [PubMed: 2577186]

22. Scher H, Herr H, Sternberg C, et al. Neo-adjuvant chemotherapy for invasive bladder cancer. Experience with the M-VAC regimen. Br J Urol. 1989; 64(3):250-256. [PubMed: 2804561]

23. Advanced Bladder Cancer Overview Collaboration. Neoadjuvant cisplatin for advanced bladder cancer. Cochrane Database Syst Rev. 2000; (2):CD001426. [PubMed: 10796791]

24. Rintala E, Hannisdahl E, Fossa SD, Hellsten S, Sander S. Neoadjuvant chemotherapy in bladder cancer: a randomized study. Nordic Cystectomy Trial I. Scand J Urol Nephrol. 1993; 27(3):355362. [PubMed: 8290916]

25. International collaboration of trialists. Neoadjuvant cisplatin, methotrexate, and vinblastine chemotherapy for muscle-invasive bladder cancer: a randomised controlled trial. Lancet. 1999; 354(9178):533-540. [PubMed: 10470696]

26. Griffiths G, Hall R, Sylvester R, Raghavan D, Parmar MK. International Phase III trial assessing neoadjuvant cisplatin, methotrexate, and vinblastine chemotherapy for muscle-invasive bladder cancer: long-term results of the BA06 30894 trial. J Clin Oncol. 2011; 29(16):2171-2177. [PubMed: 21502557]

27. Advanced Bladder Cancer Meta-analysis Collaboration. Neoadjuvant chemotherapy in invasive bladder cancer: a systematic review and meta-analysis. Lancet. 2003; 361(9373):1927-1934. [PubMed: 12801735]

28. Grossman HB, Natale RB, Tangen CM, et al. Neoadjuvant chemotherapy plus cystectomy compared with cystectomy alone for locally advanced bladder cancer. N Engl J Med. 2003; 349(9):859-866. [PubMed: 12944571]

29. Sherif A, Holmberg L, Rintala E, et al. Neoadjuvant cisplatinum based combination chemotherapy in patients with invasive bladder cancer: a combined analysis of two Nordic studies. Eur Urol. 2004; 45(3):297-303. [PubMed: 15036674] 
30. Chang JS, Lara PN Jr, Pan CX. Progress in personalizing chemotherapy for bladder cancer. Adv Urol. 2012; 2012:364919. [PubMed: 22400017]

31. Von Der Maase H, Hansen SW, Roberts JT, et al. Gemcitabine and cisplatin versus methotrexate, vinblastine, doxorubicin, and cisplatin in advanced or metastatic bladder cancer: results of a large, randomized, multinational, multicenter, Phase III study. J Clin Oncol. 2000; 18(17):3068-3077. [PubMed: 11001674]

32. Lee CT, Madii R, Daignault S, et al. Cystectomy delay more than 3 months from initial bladder cancer diagnosis results in decreased disease specific and overall survival. J Urol. 2006; 175(4): 1262-1267. discussion 1267. [PubMed: 16515975]

33. Freiha F, Reese J, Torti FM. A randomized trial of radical cystectomy versus radical cystectomy plus cisplatin, vinblastine and methotrexate chemotherapy for muscle invasive bladder cancer. $\mathrm{J}$ Urol. 1996; 155(2):495-499. discussion 499-500. [PubMed: 8558644]

34. Advanced Bladder Cancer (ABC) Meta-Analysis Collaboration. Adjuvant chemotherapy for invasive bladder cancer (individual patient data). Cochrane Database Syst Rev. 2006;

(2):CD006018. [PubMed: 16625650]

35. Millikan R, Dinney C, Swanson D, et al. Integrated therapy for locally advanced bladder cancer: final report of a randomized trial of cystectomy plus adjuvant M-VAC versus cystectomy with both preoperative and postoperative M-VAC. J Clin Oncol. 2001; 19(20):4005-4013. [PubMed: 11600601]

36. Svatek RS, Shariat SF, Lasky RE, et al. The effectiveness of off-protocol adjuvant chemotherapy for patients with urothelial carcinoma of the urinary bladder. Clin Cancer Res. 2010; 16(17):44614467. [PubMed: 20651056]

37. Loehrer PJ Sr, Einhorn LH, Elson PJ, et al. A randomized comparison of cisplatin alone or in combination with methotrexate, vinblastine, and doxorubicin in patients with metastatic urothelial carcinoma: a cooperative group study. J Clin Oncol. 1992; 10(7):1066-1073. [PubMed: 1607913]

38. Von Der Maase H. Gemcitabine in transitional cell carcinoma of the urothelium. Expert Rev Anticancer Ther. 2003; 3(1):11-19. [PubMed: 12597345]

39. Von Der Maase H, Sengelov L, Roberts JT, et al. Long-term survival results of a randomized trial comparing gemcitabine plus cisplatin, with methotrexate, vinblastine, doxorubicin, plus cisplatin in patients with bladder cancer. J Clin Oncol. 2005; 23(21):4602-4608. [PubMed: 16034041]

40. Sternberg CN, De Mulder PH, Schornagel JH, et al. Randomized Phase III trial of high-doseintensity methotrexate, vinblastine, doxorubicin, and cisplatin (MVAC) chemotherapy and recombinant human granulocyte colony-stimulating factor versus classic MVAC in advanced urothelial tract tumors: European Organization for Research and Treatment of Cancer Protocol no. 30924. J Clin Oncol. 2001; 19(10):2638-2646. [PubMed: 11352955]

41. Gallagher DJ, Bajorin DF. Neoadjuvant chemotherapy for the treatment of muscle-invasive bladder cancer: argument in favor. Nat Clin Pract Urol. 2008; 5(9):484-485. [PubMed: 18626513]

42. Fedeli U, Fedewa SA, Ward EM. Treatment of muscle invasive bladder cancer: evidence from the National Cancer Database, 2003 to 2007. J Urol. 2011; 185(1):72-78. [PubMed: 21074192]

43. Fedeli U, Novara G, Galassi C, et al. Population-based analyses of radical cystectomy and urinary diversion for bladder cancer in northern Italy. BJU Int. 2011; 108(8 Pt 2):E266-E271. [PubMed: 21320273]

44. Gandour-Edwards R, Lara PN Jr, Folkins AK, et al. Does HER2/neu expression provide prognostic information in patients with advanced urothelial carcinoma? Cancer. 2002; 95(5):1009-1015. [PubMed: 12209684]

45. Lae M, Couturier J, Oudard S, Radvanyi F, Beuzeboc P, Vieillefond A. Assessing HER2 gene amplification as a potential target for therapy in invasive urothelial bladder cancer with a standardized methodology: results in 1005 patients. Ann Oncol. 2010; 21(4):815-819. [PubMed: 19889613]

46. Stadler WM, Lerner SP, Groshen S, et al. Phase III study of molecularly targeted adjuvant therapy in locally advanced urothelial cancer of the bladder based on p53 status. J Clin Oncol. 2011; 29(25):3443-3449. [PubMed: 21810677]

47. Herr HW. Transurethral resection of muscle-invasive bladder cancer: 10-year outcome. J Clin Oncol. 2001; 19(1):89-93. [PubMed: 11134199] 
48. Herr HW, Bajorin DF, Scher HI. Neoadjuvant chemotherapy and bladder-sparing surgery for invasive bladder cancer: ten-year outcome. J Clin Oncol. 1998; 16(4):1298-1301. [PubMed: 9552029]

49. Solsona E, Climent MA, Iborra I, et al. Bladder preservation in selected patients with muscleinvasive bladder cancer by complete transurethral resection of the bladder plus systemic chemotherapy: long-term follow-up of a Phase 2 nonrandomized comparative trial with radical cystectomy. Eur Urol. 2009; 55(4):911-919. [PubMed: 18722046]

50. Rodel C, Grabenbauer GG, Kuhn R, et al. Combined-modality treatment and selective organ preservation in invasive bladder cancer: long-term results. J Clin Oncol. 2002; 20(14):3061-3071. [PubMed: 12118019]

51. Kaufman DS, Shipley WU, Griffin PP, Heney NM, Althausen AF, Efird JT. Selective bladder preservation by combination treatment of invasive bladder cancer. N Engl J Med. 1993; 329(19): 1377-1382. [PubMed: 8413433]

52. Shipley WU, Kaufman DS, Heney NM, Althausen AF, Zietman AL. An update of selective bladder preservation by combined modality therapy for invasive bladder cancer. Eur Urol. 1998; 33(Suppl 4):32-34. [PubMed: 9615208]

53. Devere White RW, Lara PN Jr, Goldman B, et al. A sequential treatment approach to myoinvasive urothelial cancer: a Phase II Southwest Oncology Group trial (S0219). J Urol. 2009; 181(6):24762480. discussion 2480-2471. [PubMed: 19371909]

54. Housset M, Maulard C, Chretien Y, et al. Combined radiation and chemotherapy for invasive transitional-cell carcinoma of the bladder: a prospective study. J Clin Oncol. 1993; 11(11):21502157. [PubMed: 8229129]

55. Vilmar A, Sorensen JB. Excision repair cross-complementation group 1 (ERCC1) in platinumbased treatment of non-small cell lung cancer with special emphasis on carboplatin: a review of current literature. Lung Cancer. 2009; 64(2):131-139. [PubMed: 18804893]

56. Vilmar AC, Santoni-Rugiu E, Sorensen JB. ERCC1 and histopathology in advanced NSCLC patients randomized in a large multicenter Phase III trial. Ann Oncol. 2010; 21(9):1817-1824. [PubMed: 20332140]

57. Bepler G, Kusmartseva I, Sharma S, et al. RRM1 modulated in vitro and in vivo efficacy of gemcitabine and platinum in non-small-cell lung cancer. J Clin Oncol. 2006; 24(29):4731-4737. [PubMed: 16966686]

58. Hoffmann AC, Wild P, Leicht C, et al. MDR1 and ERCC1 expression predict outcome of patients with locally advanced bladder cancer receiving adjuvant chemotherapy. Neoplasia. 2010; 12(8): 628-636. [PubMed: 20689757]

59. Harshman LC, Bepler G, Zheng Z, Higgins JP, Allen GI, Srinivas S. Ribonucleotide reductase subunit M1 expression in resectable, muscle-invasive urothelial cancer correlates with survival in younger patients. BJU Int. 2010; 106(11):1805-1811. [PubMed: 20438561]

60. Matsumura N, Hara I. The prognostic significance of human equilibrative nucleoside transporter1 (hENT1) expression in metastatic bladder cancer patients treated with gemcitabine-cisplatin based combination chemotherapy. Hinyokika Kiyo. 2011; 57(3):157-161. [PubMed: 21586890]

61. Takata R, Katagiri T, Kanehira M, et al. Validation study of the prediction system for clinical response of M-VAC neoadjuvant chemotherapy. Cancer Sci. 2007; 98(1):113-117. [PubMed: 17116130]

62. Williams PD, Cheon S, Havaleshko DM, et al. Concordant gene expression signatures predict clinical outcomes of cancer patients undergoing systemic therapy. Cancer Res. 2009; 69(21): 8302-8309. [PubMed: 19843853]

63. Vinall RL, Ripoll AZ, Wang S, Pan CX, Devere White RW. MiR-34a chemosensitizes bladder cancer cells to cisplatin treatment regardless of p53-Rb pathway status. Int J Cancer. 2012; 130(11):2526-2538. [PubMed: 21702042]

64. Smith SC, Havaleshko DM, Moon K, et al. Use of yeast chemigenomics and COXEN informatics in preclinical evaluation of anticancer agents. Neoplasia. 2011; 13(1):72-80. [PubMed: 21253455]

65. Henderson PT, Pan CX. Human microdosing for the prediction of patient response. Bioanalysis. 2010; 2(3):373-376. [PubMed: 21083245] 
66. Wang S, Zhang H, Malfatti M, et al. Gemcitabine causes minimal modulation of carboplatin-DNA monoadduct formation and repair in bladder cancer cells. Chem Res Toxicol. 2010; 23(11):16531655. [PubMed: 21028869]

67. Lin TY, Zhang H, Wang S, et al. Targeting canine bladder transitional cell carcinoma with a human bladder cancer-specific ligand. Mol Cancer. 2011; 10(1):9. [PubMed: 21272294]

68. Zhang H, Aina OH, Lam KS, et al. Identification of a bladder cancer-specific ligand using a combinatorial chemistry approach. Urol Oncol. 2010 (Epub ahead of print). 10.1016/j. urolonc. 2010.06.011

69. Lin T, Zhang H, Luo J, et al. Multifunctional targeting micelle nanocarriers with both imaging and therapeutic potential for bladder cancer. Int J Nanomed. 2012; 7:2793-2804.

\section{Websites}

101. ClinicalTrials.gov. Neoadjuvant Dose Dense Gemcitabine and Cisplatin (DD GC) In Patients With Muscle-Invasive Bladder Cancer. http://clinicaltrials.gov/ct2/show/NCT01589094

102. ClinicalTrials.gov. Trial of Microdosing Carboplatin. http://clinicaltrials.gov/ct2/show/ NCT01261299 


\section{Key issues}

- The prognosis for muscle-invasive bladder cancer has not changed over the last three decades.

- There is level 1 evidence supporting neoadjuvant platinum-based combination chemotherapy for bladder cancer. However, it is not widely accepted.

- Radical cystectomy is still the standard treatment for muscle-invasive bladder cancer.

- Adjuvant chemotherapy should be given to those patients who do not receive neoadjuvant chemotherapy.

- Bladder-sparing treatment can be performed in highly selected patients or patients medically unfit for radical cystectomy.

- Personalized chemotherapy targeting the underlying pathophysiological changes of urothelial carcinoma is currently being actively explored. 


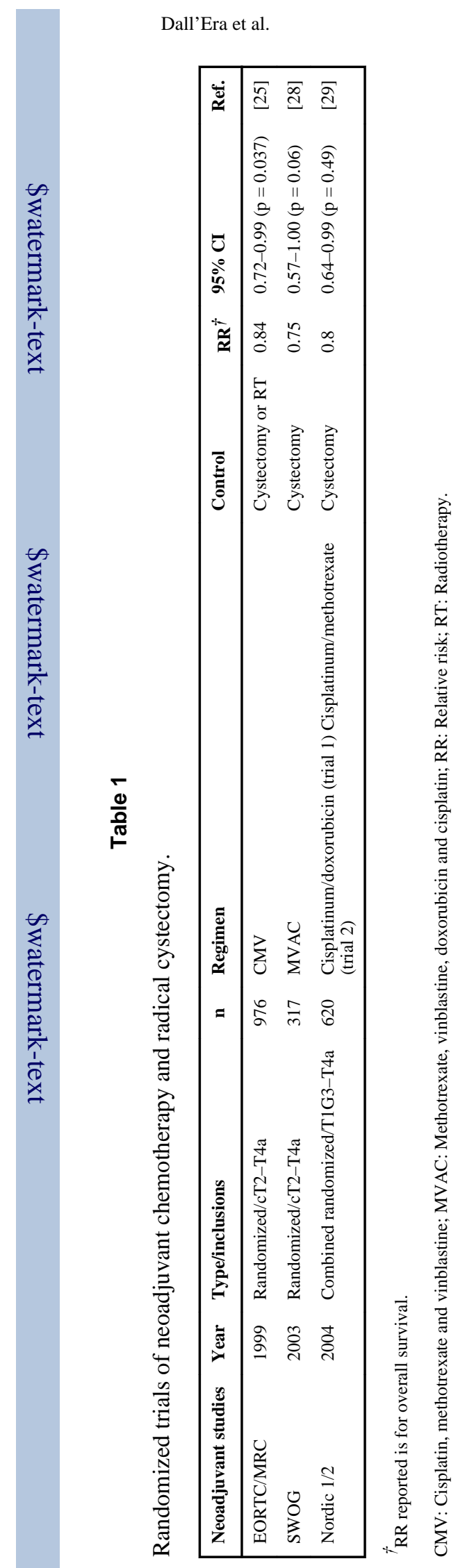

Page 16 


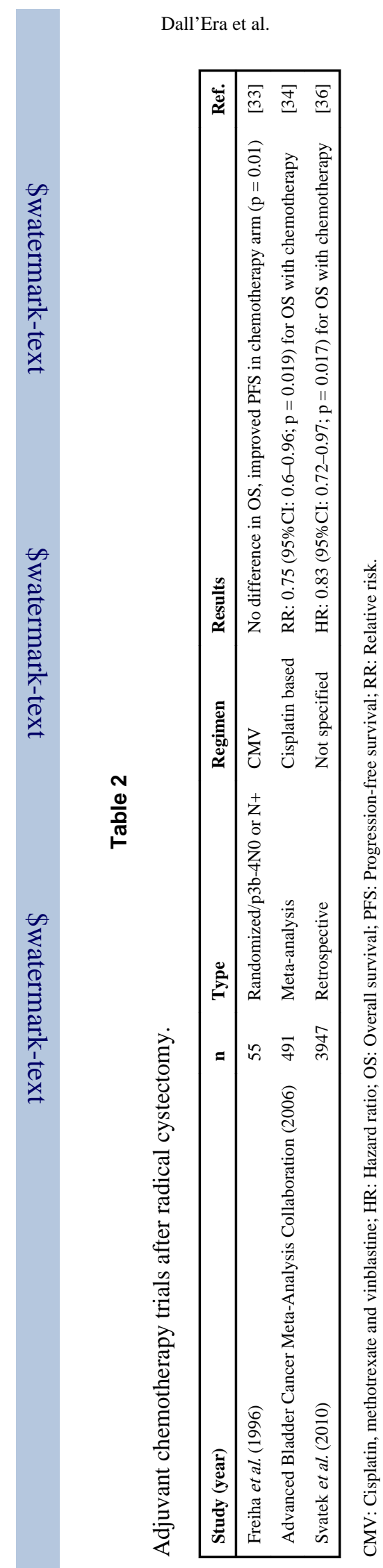

Page 17 
Table 3

Bladder preservation trials.

\begin{tabular}{|llllr|}
\hline Study (year) & n & Regimen & Results & Ref. \\
\hline Kaufman et al. (1993) & 53 & TUR/RT/chemo & OS: 45\%, 4-year median follow-up & {$[51]$} \\
Herr et al. (2001) & 99 & TUR & 10-year OS: 75\% & {$[47]$} \\
Rodel et al. (2002) & 415 & RT vs RT chemo & 10-year OS: 31\%, 80\% preserved bladders & {$[50]$} \\
Solsona et al. (2009) & 75 & TUR/chemo & 10-year CSS: 59.8\% & {$[49]$} \\
\hline
\end{tabular}

chemo: Chemotherapy; CSS: Cancer-specific survival; OS: Overall survival; RT: Radiotherapy; TUR: Transurethral resection. 